\title{
Accuracy of attenuation imaging in the assessment of pediatric hepatic steatosis: correlation with the controlled attenuation parameter
}

\author{
Pyeong Hwa Kim', Young Ah Cho', Hee Mang Yoon', Boram Bak², Jin Seong Lee', \\ Ah Young Jung ${ }^{1}$, Seak Hee $\mathrm{Oh}^{3}$, Kyung Mo $\mathrm{Kim}^{3}$ \\ 'Department of Radiology and Research Institute of Radiology, Asan Medical Center, \\ University of Ulsan College of Medicine, Seoul; ${ }^{2}$ University of Ulsan Foundation for Industry \\ Cooperation, Ulsan; ${ }^{3}$ Department of Pediatrics, Asan Medical Center, University of Ulsan \\ College of Medicine, Seoul, Korea
}

Purpose: This study evaluated the accuracy of attenuation imaging (ATI) for the assessment of hepatic steatosis in pediatric patients, in comparison with the FibroScan vibration-controlled transient elastography controlled attenuation parameter (CAP).

Methods: Consecutive pediatric patients referred for evaluation of obesity who underwent both ATI and FibroScan between February 2020 and September 2021 were included. The correlation between attenuation coefficient $(A C)$ and CAP values was assessed using the Spearman test. The AC cutoff value for discriminating hepatic steatosis corresponding to a CAP value of $241 \mathrm{~dB} / \mathrm{m}$ was calculated. Multivariable linear regression analysis was performed to estimate the strength of the association between AC and CAP. The diagnostic accuracy of AC cutoffs was estimated using the imperfect gold-standard methodology based on a two-level Bayesian latent class model.

Results: Seventy patients (median age, 12.5 years; interquartile range, 11.0 to 14.0 years; male:female, 58:12) were included. AC and CAP showed a moderate-to-good correlation ( $\rho$ $=0.646, P<0.001)$. Multivariable regression analysis affirmed the significant association between $A C$ and CAP $(P<0.001)$. The correlation was not evident in patients with a body mass index $\geq 30$ $\mathrm{kg} / \mathrm{m}^{2}(\rho=-0.202, P=0.551)$. Linear regression revealed that an $A C$ cutoff of $0.66 \mathrm{~dB} / \mathrm{cm} / \mathrm{MHz}$ corresponded to a CAP of $241 \mathrm{~dB} / \mathrm{m}$ (sensitivity, $0.93 ; 95 \%$ confidence interval [CI], 0.85 to 0.98 and specificity, $0.87 ; 95 \% \mathrm{Cl}, 0.56$ to 1.00$)$.

Conclusion: ATI showed an acceptable correlation with CAP values in a pediatric population, especially in patients with a body mass index $<30 \mathrm{~kg} / \mathrm{m}^{2}$. An AC cutoff of $0.66 \mathrm{~dB} / \mathrm{cm} / \mathrm{MHz}$, corresponding to a CAP of $241 \mathrm{~dB} / \mathrm{m}$, can accurately diagnose hepatic steatosis.

Keywords: Pediatrics; Obesity; Fatty liver; Nonalcoholic fatty liver disease; Ultrasonography Key points: A moderate-to-good correlation was found between the attenuation coefficient (AC) and controlled attenuation parameter $(\rho=0.646)$, as confirmed by multivariable regression analysis. There was a poor correlation between $A C$ and controlled attenuation parameter in patients with a body mass index $\geq 30 \mathrm{~kg} / \mathrm{m}^{2}(\rho=-0.202)$. The sensitivity and specificity of $A C$ with a cutoff of $0.66 \mathrm{~dB} / \mathrm{cm} / \mathrm{MHz}$ based on a two-level Bayesian latent class model were 0.93 (95\% confidence interval $[\mathrm{Cl}], 0.85$ to 0.98$)$ and $0.87(95 \% \mathrm{Cl}, 0.56$ to 1.00$)$, respectively.

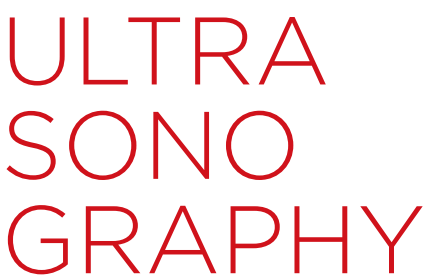

\section{ORIGINAL ARTICLE}

https://doi.org/10.14366/usg.21246 pISSN: 2288-5919 - elSSN: 2288-5943 Ultrasonography 2022;41:761-769

Received: November 24, 2021

Revised: February 24, 2022

Accepted: March 5, 2022

Correspondence to:

Young Ah Cho, MD, PhD, Department of Radiology and Research Institute of Radiology, University of Ulsan College of Medicine, Asan Medical Center, 88 Olympic-ro 43-gil, Songpa-gu, Seoul 05505, Korea

Tel. +82-2-3010-5763

Fax. +82-2-476-0090

E-mail: ped.yacho@gmail.com

This is an Open Access article distributed under the terms of the Creative Commons Attribution NonCommercial License (http://creativecommons.org/ licenses/by-nc/4.0/) which permits unrestricted noncommercial use, distribution, and reproduction in any medium, provided the original work is properly cited.

Copyright (C) 2022 Korean Society of Ultrasound in Medicine (KSUM)

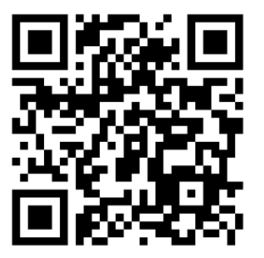

How to cite this article: Kim PH, Cho YA, Yoon HM, Bak B, Lee JS, Jung AY, et al. Accuracy of attenuation imaging in the assessment of pediatric hepatic steatosis: correlation with the controlled attenuation parameter. Ultrasonography. 2022 Oct;41(4):761-769. 


\section{Introduction}

Nonalcoholic fatty liver disease (NAFLD) is common in pediatric populations, and fatty liver is the most common liver disease in children and adolescents in the United States, being present in 13\% of subjects in autopsy studies [1]. It is more common in Asians, boys, obese patients, and patients with prediabetes or diabetes [1-3]. As children with NAFLD have a higher risk of end-stage liver disease [4], cardiovascular disease [5], and hepatocellular carcinoma $[6,7]$, it is obvious that early diagnosis and treatment of pediatric NAFLD are important issues.

Currently, liver biopsy is the most reliable reference standard for the diagnosis of fatty liver, and beyond its role in diagnostic confirmation, it also provides useful information on the degree of liver damage, architectural change, and fibrosis [3]. However, liver biopsy is not practical for screening purposes because of its invasive nature. Various noninvasive imaging modalities, including ultrasonography (US), computed tomography (CT), and magnetic resonance imaging (MRI), have been used to provide alternative methods for the diagnosis of fatty liver [8]. Among them, the FibroScan (Echosens, Paris, France) vibration-controlled transient elastography controlled attenuation parameter (CAP), which enables quantification of hepatic steatosis by measuring the ultrasonic attenuation of the echo wave [9], is widely used because of its noninvasive and reproducible characteristics. The diagnostic accuracy of CAP is well-validated in adults [10] and also relatively wellvalidated in pediatric populations $[11,12]$. However, it has several disadvantages: (1) it requires a separate FibroScan machine in addition to a general US scanner; (2) CAP is measured in a blind fashion and inevitably includes large vessels, focal hepatic lesions, and ascites; and (3) CAP is affected by abdominal wall thickness [13].

In recent studies, attenuation imaging (ATI), a two-dimensional US-based fat-quantification technique that measures the attenuation coefficient (AC) within a region of interest (ROI) placed on a grayscale US image, has shown acceptable accuracy in the detection of fatty liver in adult populations [14-16]. As ATI enables hepatic fat quantification during a single-sitting US examination, it has a potential role in pediatric patients. However, its diagnostic accuracy has not been clearly validated in a pediatric population. Therefore, the aim of this study was to evaluate the accuracy of ATI in the assessment of hepatic steatosis in a pediatric population by evaluating its correlation with CAP.

\section{Materials and Methods}

\section{Compliance with Ethical Standards}

This retrospective observational study was conducted at a single tertiary hospital and was approved by the Institutional Review Board (2021-1663). The requirement for informed consent was waived. This retrospective study was conducted in accordance with Strengthening the Reporting of Observational Studies in Epidemiology (STROBE) guidelines [17].

\section{Patient Selection and Clinical Data Collection}

Consecutive pediatric patients (age $\leq 18$ years) who were referred to the authors' institution for the evaluation of fatty liver, and who underwent both ATI and FibroScan between February 2020 and September 2021, were included. At the authors' institution, the diagnostic work-up for NAFLD (including FibroScan and US) was generally indicated in patients with abnormal alanine aminotransferase (ALT) levels (e.g., persistently [>3 months] elevated ALT higher than cutoff value of $52 \mathrm{IU} / \mathrm{L}$ for boys and 44 IU/L for girls, or ALT higher than $80 \mathrm{IU} / \mathrm{L}$ ) with/without body mass index (BMI) higher than the 85th percentile. This work-up was also indicated for patients who showed sudden weight gain regardless of BMI, or incidentally detected imaging findings suspicious of fatty liver (e.g., diffuse increase in echogenicity on US; diffuse decrease in attenuation on (T). ATI was performed in addition to grayscale US if the liver showed high echogenicity resulting in a suspicion of fatty liver. Patients who met any of the following criteria were excluded: (1) an interval between ATI and FibroScan of $>7$ days; (2) liver transplantation; and (3) confirmation of disease other than fatty liver that might affect the liver (e.g., biliary atresia, metabolic disease, or cardiac disease). None of the patients included in this study were previously reported.

Clinical data including sex, age at examination, BMI, and levels of aspartate transaminase (AST), ALT, gamma-glutamyl transferase ( $r$ GT), fasting glucose, triglycerides, low-density lipoprotein (LDL), and high-density lipoprotein (HDL) were collected.

\section{Controlled Attenuation Parameter}

The CAP, which is based on the ultrasonic properties of the backpropagated radiofrequency signals acquired by the FibroScan system [18], was measured by one of three trained radiologists (P.H.K., H.M.Y., and Y.A.C.; with 5, 12, and 27 years of experience in radiology, respectively). The radiologists were blinded to the clinical data except for age and sex. All measurements were performed using the $\mathrm{M}$ probe $(3.5 \mathrm{MHz}$ ) of a FibroScan-502 system. Patients were asked to fast for at least 3 hours before the examination. The probe was placed in the intercostal space, and CAP was measured in the right liver. During the examinations, patients were instructed to lie in the supine position with their right arm raised. The procedure was deemed to be valid only when measurements were obtained at least 10 times. 


\section{Attenuation Imaging}

The ATI-based AC was routinely measured in patients suspected to have NAFLD by one of three trained radiologists using an Aplio i800 US machine (Canon Medical Systems, Otawara, Japan) using an $\mathrm{i} 8 \mathrm{CX} 1$ transducer. It is well known that the US beam is more attenuated by intracellular fat vacuoles than by normal liver tissue, resulting in an increase of the liver parenchymal echogenicity in fatty liver. In contrast to CAP, which is a one-dimensional attenuation based quantification technique from transient elastography, ATI is a two-dimensional attenuation technique based on B-mode US images. It enables quantification of the degree of loss of US energy passing through the liver by calculating the AC. For an accurate measurement of the $A C$, the systemic influences from the beam profile and internal gain control are automatically removed. The AC then can be calculated as the slope of the line profile [19].

Before the US examination, patients were asked to fast for at least 3 hours and to lie in the supine position with their right arm raised. Grayscale US was initially performed to find the optimal location for observing the liver parenchyma, and then the ATI mode was activated. Patients were asked to hold their breath during the examination, if possible. The sampling was set as wide as possible, and the ROI was placed at least $2 \mathrm{~cm}$ below the skin to avoid reverberation artifacts. Of note, structures other than liver parenchyma, such as vessels, were automatically excluded from the ATI measurements on the basis of their intensity variance. When the $\mathrm{ROI}$ was placed in an appropriate position, the AC value and an $R^{2}$ value indicating the reliability of the result were displayed. The ATI measurement was deemed to be appropriate when $R^{2}$ was 0.80 or more, and the measurement was deemed to be valid only when appropriate measurements were obtained at least five times [19].

\section{Outcomes and Statistical Analysis}

The Spearman correlation test was used to estimate the correlation between $A C$ and $C A P$, using the median of five appropriately measured $A C$ values. In addition, the strength of the association between $A C$ and CAP was evaluated using multivariable linear regression analysis. In detail, univariable linear regression analysis was first performed to explore potential factors associated with CAP. The covariates considered in this regression analysis, which included AC, were as follows: age, sex, BMI, obesity (normal, BMI $<85$ th percentile; overweight, BMI 85th-94th percentile; obesity, BMI 95th-98th percentile; severe obesity, BMI $\geq 99$ th percentile; according to 2019 Korean Society of Pediatric Gastroenterology Hepatology and Nutrition clinical practice guideline [20]) and levels of ALT, AST, r-GT, fasting glucose, triglycerides, LDL, and HDL.

Multivariable linear regression analysis was then performed to calculate the adjusted regression coefficient and P-value of $A C$, including the covariates that showed significant correlations with CAP in the univariable analysis $(P<0.05)$ and ALT, which is a wellknown risk factor for fatty liver [21].

A subgroup analysis was also performed to estimate the degree of correlation between CAP and AC within specific subgroups. In this analysis, the study population was grouped not only according to the covariates showing significant correlations with CAP in the linear regression analysis (i.e., sex and obesity), but also according to BMI (with a cutoff value of $30 \mathrm{~kg} / \mathrm{m}^{2}$ ) and ALT. The BMI cutoff of $30 \mathrm{~kg} / \mathrm{m}^{2}$ was used for the subgroup analysis because a previous study found that pediatric patients with a BMI $\geq 30 \mathrm{~kg} / \mathrm{m}^{2}$ showed a poor correlation between CAP and MRI proton density fat fraction (PDFF) [12]. Patients were dichotomized using an ALT cutoff value of $52 \mathrm{IU} / \mathrm{L}$ for boys and $44 \mathrm{IU} / \mathrm{L}$ for girls, which are the suggested cutoff values for discriminating fatty liver [21].

A linear regression model was also used to calculate an AC cutoff value for the diagnosis of fatty liver by determining the $A C$ value corresponding to a CAP value of $241 \mathrm{~dB} / \mathrm{m}$, which is the suggested cutoff value for discriminating fatty liver in the Korean pediatric population [12]. In this cited study, the reference standard was MRIPDFF, with the PDFF cutoff values being $6 \%$ for histologic steatosis grade 1 (S1), $17.5 \%$ for grade 2 (S2), and $23.3 \%$ for grade 3 (S3) [22]. Of note, the histologic steatosis grade is determined according to the proportion of hepatocytes containing fat macrovesicles: $\mathrm{SO}$ for $<5 \%$, S1 for $5 \%-33 \%$, S2 for $34 \%-66 \%$, and S3 for $>66 \%$ [23]. Furthermore, AC cutoffs corresponding to $299 \mathrm{~dB} / \mathrm{m}$ (the suggested CAP cutoff value for discriminating S0-1 from S2-3 based on MRIPDFF as the reference standard) and $303 \mathrm{~dB} / \mathrm{m}$ (the suggested CAP cutoff for discriminating S0-2 from S3 based on MRI-PDFF as the reference standard) were also calculated [12].

The diagnostic accuracies of CAP, AC, obesity (BMI 95th-98th percentile) or severe obesity (BMI $\geq 99$ th percentile), and ALT cutoffs were estimated using the imperfect gold-standard methodology based on a two-level Bayesian latent class model [24], as previously reported $[11,25]$. Briefly, the latent class model analysis combines different index tests, all of which are influenced by the unknown true status (disease-positive or disease-negative), which is treated as the latent variable. In this Bayesian approach, disease prevalence is incorporated into the model, and unknown parameters are treated as random variables with a probability distribution, rather than as fixed unknown quantities. The diagnostic accuracies of ATI for discrimination of S0-1 from S2-3 and S0-2 from S3 were not calculated because the cutoff values for BMI and ALT are not clearly established.

As a rule of thumb, the Spearman correlation coefficient is interpreted as follows: $0-0.25$, little or no correlation; $0.25-0.50$, a fair degree of correlation; $0.50-0.75$, moderate-to-good correlation; 
and $0.75-1.00$, very good to excellent correlation [26]. The twolevel Bayesian latent class model used to estimate diagnostic accuracy was constructed using a web-based tool (http://mice. tropmedres.ac/home.aspx), whereas the other statistical analyses were conducted using $R$ version 3.1.2 (R Foundation for Statistical Computing, Vienna, Austria). A two-sided P-value of $<0.05$ was considered statistically significant.

\section{Results}

\section{Patients}

During the study period, 91 pediatric patients underwent abdominal

Table 1. Demographics and clinical characteristics of the 70 included patients

\begin{tabular}{lc}
\hline \multicolumn{1}{c}{ Characteristic } & Value \\
\hline Age (year) & $12.5(11.0-14.0)$ \\
Sex & \\
Male & $58(82.9)$ \\
Female & $12(17.1)$ \\
BMI (kg/m $\left.{ }^{2}\right)$ & $26.4 \pm 3.8$ \\
$<25$ & $22(31.4)$ \\
$25-30$ & $37(52.9)$ \\
$\geq 30$ & $11(15.7)$ \\
BMI percentile & \\
Normal (<85th percentile) & $10(14.3)$ \\
Overweight (85th-94th percentile) & $14(20.0)$ \\
Obesity (95th-98th percentile) & $6(8.6)$ \\
Severe obesity ( $\geq 99$ th percentile) & $40(57.1)$ \\
ALT (IU/L) & $106.3 \pm 84.9$ \\
Normal & $22(31.4)$ \\
Abnormal) & $48(68.6)$ \\
AST (IU/L) & $57.2 \pm 36.8$ \\
r-GT (IU/L) & $49.7 \pm 30.6$ \\
Fasting glucose (mg/dL) & $97.2 \pm 9.8$ \\
Triglycerides (mg/dL) & $133.1 \pm 60.1$ \\
LDL (mg/dL) & $124.3 \pm 26.2$ \\
HDL (mg/dL) & $46.1 \pm 9.2$ \\
CAP (dB/m) & $295.8 \pm 43.3$ \\
$<241$ & $9(12.9)$ \\
$\geq 241$ & $61(87.1)$ \\
\hline & \\
\hline &
\end{tabular}

Values are presented as median (IQR), number (\%), or mean $\pm S D$.

$B M I$, body mass index; ALT, alanine transaminase; AST, aspartate transaminase; r-GT, gamma-glutamyl transferase; LDL, low-density lipoprotein; HDL, high-density lipoprotein; CAP, controlled attenuation parameter; IQR, interquartile range; SD, standard deviation.

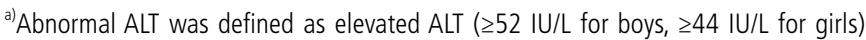
more than twice the upper limit of normal (26 IU/L for boys, $22 \mathrm{IU} / \mathrm{L}$ for girls).
US for the evaluation of fatty liver. Of these, 20 patients did not undergo ATI since the Aplio i800 US machine providing ATI function was not available at the time of the US exam (instead, the other US machine, which did not provide ATI, was used for grayscale US). Thus, 71 patients underwent both FibroScan and ATI, although in one patient the interval between FibroScan and ATI was longer than 7 days. Consequently, a total of 70 patients (median age, 12.5 years; interquartile range, 11.0 to 14.0 years; male:female, 58:12) were included in the analysis. There was no case of technical failure with either FibroScan or ATI. The interquartile range and median values of AC did not show statistically significant differences depending on BMI (mean \pm standard deviation: $<25 \mathrm{~kg} / \mathrm{m}^{2}, 0.08 \pm 0.04 ; 25-30 \mathrm{~kg} /$ $\mathrm{m}^{2}, 0.06 \pm 0.04 ; \geq 30 \mathrm{~kg} / \mathrm{m}^{2}, 0.06 \pm 0.02 \mathrm{~dB} / \mathrm{cm} / \mathrm{MHz} ; \mathrm{P}=0.280$ ) or $\mathrm{BMl}$ percentile (mean \pm standard deviation: normal, $0.08 \pm 0.04$; overweight, $0.07 \pm 0.04$; obesity, $0.06 \pm 0.02$; severe obesity, $0.06 \pm 0.04 \mathrm{~dB} / \mathrm{cm} /$ $\mathrm{MHz} ; \mathrm{P}=0.371)$. The detailed demographics and clinical characteristics of the included patients are summarized in Table 1.

\section{Correlation between CAP and ATI}

The Spearman correlation test demonstrated a good correlation between CAP and AC $(\rho=0.646 . P<0.001)$. The univariable regression analysis also showed a significant association, with the following regression equation being estimated: $A C=0.001925 \times C A P+0.195454$ (Fig. 1). The multivariable regression

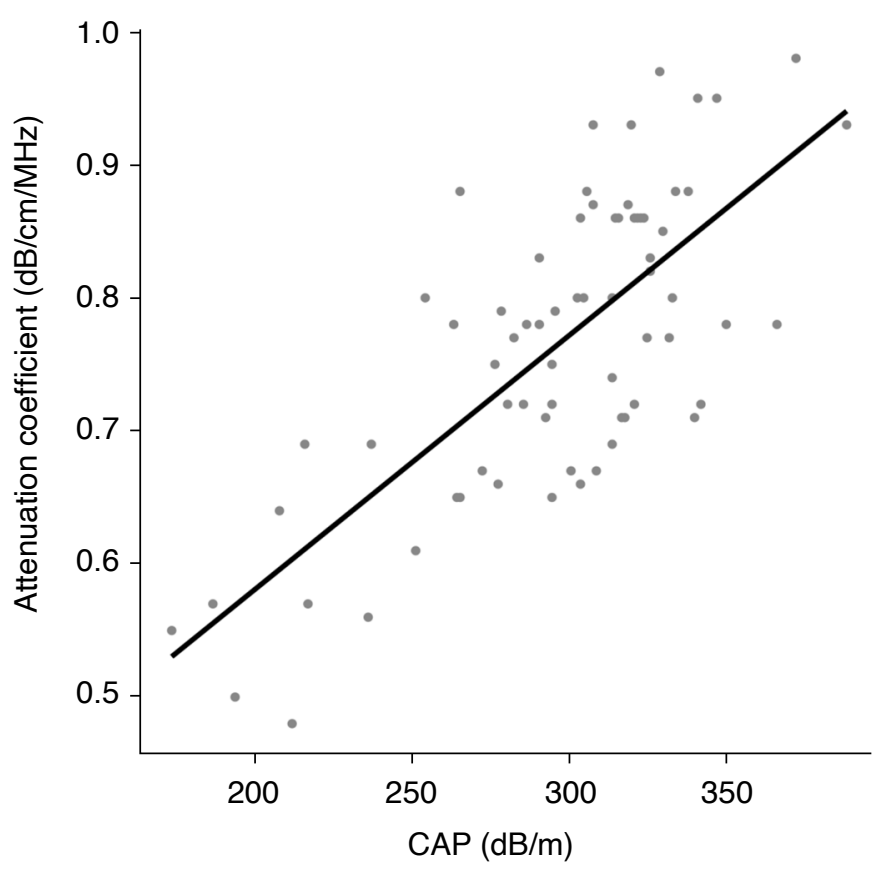

Fig. 1. Scatterplot with linear regression line for the attenuation coefficient in relation to the controlled attenuation parameter (CAP). There was a moderate-to-good correlation between the attenuation coefficient and $\operatorname{CAP}(\rho=0.646, P<0.001)$. 
analysis, which included sex (as a variable that showed a significant association with CAP in the univariable analysis), obesity, and ALT as covariates, also showed a significant association between CAP and AC $(\mathrm{P}<0.001)$ (Table 2).

The results of the subgroup analyses are summarized in Table 3. Significant correlations between CAP and AC were still found when the patients were divided into subgroups according to sex and ALT. However, when the patients were dichotomized according to BMI, a correlation between CAP and AC was not evident in patients with a BMI $\geq 30 \mathrm{~kg} / \mathrm{m}^{2}$ ( $\left.\rho=-0.202, P=0.551\right)$. Indeed, when those patients were excluded, the Spearman rank correlation coefficient in patients with a $\mathrm{BMl}<30 \mathrm{~kg} / \mathrm{m}^{2}$ increased to $0.730(\mathrm{P}<0.001)$.

\section{Diagnostic Accuracy}

According to the univariable linear regression model for the relationship between $A C$ and $C A P$, the $A C$ value corresponding to $241 \mathrm{~dB} / \mathrm{m}$ (the suggested CAP cutoff for the diagnosis of fatty liver) was $0.66 \mathrm{~dB} / \mathrm{cm} / \mathrm{MHz}$. In addition, the $A C$ values corresponding to $299 \mathrm{~dB} / \mathrm{m}$ (the suggested CAP cutoff for discriminating S0-1 from $\mathrm{S} 2-3$ ) and $303 \mathrm{~dB} / \mathrm{m}$ (the suggested CAP cutoff for discriminating S0-2 from S3) were 0.77 and $0.78 \mathrm{~dB} / \mathrm{cm} / \mathrm{MHz}$, respectively. When an $A C$ value of $0.66 \mathrm{~dB} / \mathrm{cm} / \mathrm{MHz}$ was adopted as the cutoff value for the diagnosis of fatty liver, the sensitivity and specificity of $A C$ were 0.93 (95\% confidence interval [CI], 0.85 to 0.98$)$ and $0.87(95 \% \mathrm{Cl}$, 0.56 to 1.00 ), respectively (Table 4). Regarding the other variables, the sensitivity and specificity were $0.99(95 \% \mathrm{Cl}, 0.95$ to 1.00$)$ and $1.00(1.00$ to 1.00$)$ for CAP, $0.69(95 \% \mathrm{Cl}, 0.56$ to 0.79$)$ and

Table 2. Multivariable linear regression analysis assessing the strength of the association between the attenuation coefficient and controlled attenuation parameter

\begin{tabular}{|c|c|c|c|c|c|c|}
\hline \multirow[b]{2}{*}{ Variable } & \multicolumn{3}{|c|}{ Univariable analysis } & \multicolumn{3}{|c|}{ Multivariable analysis } \\
\hline & $\begin{array}{l}\text { Regression coefficient } \\
\text { (standard error) }\end{array}$ & $95 \% \mathrm{Cl}$ & P-value & $\begin{array}{l}\text { Regression coefficient } \\
\text { (standard error) }\end{array}$ & $95 \% \mathrm{Cl}$ & P-value \\
\hline \multicolumn{7}{|l|}{ Main factor } \\
\hline $\begin{array}{l}\text { Attenuation coefficient } \\
(\mathrm{dB} / \mathrm{cm} / \mathrm{MHz})\end{array}$ & $279.900(31.410)$ & 217.231 to 342.573 & $<0.001$ & $299.815(33.717)$ & 232.437 to 367.192 & $<0.001$ \\
\hline \multicolumn{7}{|l|}{ Covariates } \\
\hline Age (year) & $3.235(1.963)$ & -0.680 to 7.151 & 0.104 & & & \\
\hline \multicolumn{7}{|l|}{ Sex } \\
\hline Female & Reference category & & & Reference category & & \\
\hline Male & $27.240(13.420)$ & 0.455 to 54.016 & 0.046 & $23.527(9.652)$ & 4.240 to 42.814 & 0.018 \\
\hline $\mathrm{BMI}\left(\mathrm{kg} / \mathrm{m}^{2}\right)$ & $1.982(1.373)$ & -0.758 to 4.721 & 0.153 & & & \\
\hline$<30 \mathrm{~kg} / \mathrm{m}^{2}$ & Reference category & & & & & \\
\hline$\geq 30 \mathrm{~kg} / \mathrm{m}^{2}$ & $21.049(13.721)$ & -6.339 to 48.437 & 0.130 & & & \\
\hline \multicolumn{7}{|l|}{ BMI percentile } \\
\hline $\begin{array}{l}\text { Normal }(<85 \text { th } \\
\text { percentile) }\end{array}$ & Reference category & & & Reference category & & \\
\hline $\begin{array}{l}\text { Overweight (85th- } \\
\text { 94th percentile) }\end{array}$ & $26.910(17.980)$ & -8.991 to 62.819 & 0.139 & $-3.691(12.429)$ & -28.529 to 21.147 & 0.768 \\
\hline $\begin{array}{l}\text { Obesity (95th-98th } \\
\text { percentile) }\end{array}$ & $25.030(22.430)$ & -19.748 to 69.815 & 0.268 & $-1.583(15.198)$ & -31.953 to 28.787 & 0.917 \\
\hline $\begin{array}{l}\text { Severe obesity ( } \geq 99 \text { th } \\
\text { percentile) }\end{array}$ & $17.630(15.360)$ & -13.035 to 48.285 & 0.255 & $14.952(10.494)$ & -6.018 to 35.922 & 0.159 \\
\hline $\mathrm{ALT}(\mathrm{IU} / \mathrm{L})$ & $0.085(0.061)$ & -0.036 to 0.207 & 0.166 & $-0.066(0.044)$ & -0.155 to 0.023 & 0.144 \\
\hline AST (IU/L) & $0.102(0.142)$ & -0.182 to 0.385 & 0.476 & & & \\
\hline r-GT (IU/L) & $0.199(0.170)$ & -0.145 to 0.542 & 0.249 & & & \\
\hline Fasting glucose (mg/dL) & $0.550(0.534)$ & -0.516 to 1.616 & 0.307 & & & \\
\hline Triglycerides (mg/dL) & $0.026(0.091)$ & -0.155 to 0.208 & 0.773 & & & \\
\hline LDL (mg/dL) & $-0.042(0.214)$ & -0.471 to 0.387 & 0.845 & & & \\
\hline $\mathrm{HDL}(\mathrm{mg} / \mathrm{dL})$ & $-0.028(0.596)$ & -1.219 to 1.162 & 0.962 & & & \\
\hline
\end{tabular}

$\mathrm{Cl}$, confidence interval; BMI, body mass index; AST, aspartate transaminase; ALT, alanine transaminase; r-GT, gamma-glutamyl transferase; LDL, low-density lipoprotein; HDL, high-density lipoprotein. 
$0.63(95 \% \mathrm{Cl}, 0.42$ to 0.88$)$ for obesity or severe obesity, and 0.75 ( $95 \% \mathrm{Cl}, 0.63$ to 0.85$)$ and $0.86(95 \% \mathrm{Cl}, 0.55$ to 0.99$)$ for ALT, respectively.

\section{Discussion}

The present study demonstrated that ATI is a good alternative to the FibroScan vibration-controlled transient elastography CAP measurement for the diagnosis of fatty liver in a pediatric population. A moderate-to-good correlation was found between $A C$ and $C A P$, as confirmed by multivariable regression analysis. In addition, ATI with an AC cutoff value of $0.66 \mathrm{~dB} / \mathrm{cm} / \mathrm{MHz}$ was accurate for the diagnosis of fatty liver.

The diagnostic performance of ATI in a pediatric population in this study was comparable to the values reported in adult populations $[15,16,27,28]$. A previous study that included 48 pediatric

Table 3. Subgroup analysis of the correlation between the attenuation coefficient and controlled attenuation parameter

\begin{tabular}{lcr}
\hline \multicolumn{1}{c}{ Subgroup } & $\begin{array}{c}\text { Spearman correlation } \\
\text { coefficient }\end{array}$ & P-value \\
\hline Sex & 0.779 & 0.003 \\
Female & 0.610 & $<0.001$ \\
Male & & \\
BMI (kg/m $\left.{ }^{2}\right)$ & 0.730 & $<0.001$ \\
$<30$ & -0.202 & 0.551 \\
$\geq 30$ & & \\
BMI percentile & 0.772 & 0.009 \\
Normal (<85th percentile) & 0.738 & 0.003 \\
Overweight (85th-94th percentile) & 0.912 & 0.011 \\
Obesity (95th-98th percentile) & 0.544 & $<0.001$ \\
Severe obesity ( $\geq 99$ th percentile) & & \\
ALT & 0.850 & 0.007 \\
Normal & 0.548 & $<0.001$ \\
Abnormal & & \\
\hline
\end{tabular}

BMI, body mass index; ALT, alanine transaminase.

${ }^{\text {a) }}$ Abnormal ALT was defined as elevated ALT ( $\geq 52$ IU/L for boys, $\geq 44$ IU/L for girls) more than twice the upper limit of normal (26 IU/L for boys, 22 IU/L for girls). patients also showed that AC had good diagnostic performance in comparison with a reference standard of MRI-PDFF, with a sensitivity of 0.80 and a specificity of 0.98 using an $A C$ threshold of $0.60 \mathrm{~dB} /$ $\mathrm{cm} / \mathrm{MHz}$ [29]. In a meta-analysis of adult populations, the pooled sensitivity and specificity of AC were 0.76 and 0.84 , respectively [28].

Compared with the imaging modalities available for adults, those available for diagnosing fatty liver in children are limited. Although CT is easy to perform and widely used in adults, it should be cautiously used in children because of its relative radiation hazard $[30,31]$. MRI is a valuable noninvasive imaging tool for the detection and quantification of liver fat and does not pose a risk of exposure to ionizing radiation. However, sedation is generally required for young children. Furthermore, MRI is expensive and requires dedicated sequences for fat quantification. Liver biopsy is, needless to say, invasive and unsuitable for screening purposes. By contrast, US is a simple radiation-free inexpensive modality. Considering this, it is important to demonstrate the diagnostic performance of ATI for fatty liver.

ATI has several advantages over FibroScan CAP. First, ATI can be performed in one sitting along with the main abdominal US examination, whereas CAP can only be obtained using a FibroScan machine, which does not provide any grayscale imaging. Second, the ROI for ATI measurement can be placed in an appropriate area, avoiding unreliable structures and lesions such as hepatic vessels, biliary tree, gallbladder, and ascites. Third, ATI can measure AC differently in cases of uneven hepatic fat infiltration. Last, because of its inexpensiveness and simplicity, ATI is suitable for repetitive monitoring.

In addition, AC is theoretically less affected by tissue outside of the $\mathrm{ROI}$ because $A C$ is estimated by calculating the spatial rate of ultrasound energy loss in tissue limited to the ROI [19]. Bae et al. [15] also reported that $A C$ was not statistically associated with the skinto-liver capsule distance in their multivariable analysis. Furthermore, the intra-observer reliability of $A C$ was reported to be excellent independently of the skin-to-liver capsule distance, although a sensitivity analysis of interobserver reliability was not available [32]. In contrast, it has been reported that CAP has a positive correlation with abdominal wall thickness [12]. In the present study, there

Table 4. Diagnostic accuracy of the dichotomized CAP, ATI, obesity or severe obesity, and ALT in the diagnosis of fatty liver

\begin{tabular}{llccc}
\hline Index test & \multicolumn{1}{c}{ Cutoff value } & Sensitivity $(95 \% \mathrm{Cl})$ & Specificity $(95 \% \mathrm{Cl})$ & Accuracy $(95 \% \mathrm{Cl})$ \\
\hline CAP & $241 \mathrm{~dB} / \mathrm{m}$ & $0.99(0.95-1.00)$ & $1.00(1.00-1.00)$ & $0.99(0.92-1.00)$ \\
ATI & $0.66 \mathrm{~dB} / \mathrm{cm} / \mathrm{MHz}$ & $0.93(0.85-0.98)$ & $0.87(0.56-1.00)$ & $0.93(0.84-0.97)$ \\
Obesity or severe obesity & $\mathrm{BMI} \geq 95$ th percentile & $0.69(0.56-0.79)$ & $0.63(0.42-0.88)$ & $0.69(0.57-0.78)$ \\
ALT & $52 \mathrm{IU} / \mathrm{L}$ for boys, 44 IU/L for girls & $0.75(0.63-0.85)$ & $0.86(0.55-0.99)$ & $0.76(0.64-0.84)$ \\
\hline
\end{tabular}

Diagnostic accuracy was estimated using the imperfect gold-standard methodology based on a two-level Bayesian latent class model. CAP, controlled attenuation parameter; ATI, attenuation imaging; ALT, alanine aminotransferase; $\mathrm{Cl}$, confidence interval. 
was a poor correlation between CAP and ATI in patients with a $B M I \geq 30 \mathrm{~kg} / \mathrm{m}^{2}$; indeed, FibroScan measurement failure was more frequently observed in obese patients, especially when using the $M$ probe $[33,34]$. Furthermore, CAP was overestimated in patients with a large skin-to-capsular distance, leading to inferior diagnostic accuracy for the diagnosis of hepatic steatosis [35]. In addition, one study showed that interobserver agreement was inversely correlated with BMI [36]. As obesity has an apparent correlation with NAFLD [37], patients with suspected fatty liver tend to be obese and have a thick abdominal wall. Therefore, ATI may be more accurate in very obese patients. However, further research is necessary to clarify this point.

The present study has several limitations of note. First, the number of included patients was modest, limiting the statistical power. Second, a robust reference standard such as liver biopsy or MRI was not obtained. Instead, this study used the imperfect gold-standard methodology based on a two-level Bayesian latent class model. However, this method generates results for the "relative" sensitivity and specificity in comparison with other index tests included in the model [38]. Considering that FibroScan CAP is not a perfect diagnostic test, the calculated sensitivity and specificity of ATI would be inevitably overestimated. Further validation studies with the use of a robust standard are mandatory. Third, the diagnostic performance of ATI for detection of moderate-to-severe or severe degree of fatty liver was not determined. Fourth, an intra- or interreader reliability analysis was not conducted. Measurements may be particularly difficult in children because of their irregular breathing and poor cooperation. Further research with a larger sample size and a robust reference standard seems mandatory to clarify these issues. Fifth, although the persistence of elevated ALT levels ( $>3$ months) is emphasized for the screening of pediatric fatty liver, only the ALT values at the time of US exams were used in the present study due to the unavailability of previous ALT values in general.

In conclusion, ATI showed an acceptable correlation with CAP values in a pediatric population, especially in patients with a BMI $<30 \mathrm{~kg} / \mathrm{m}^{2}$. An AC cutoff of $0.66 \mathrm{~dB} / \mathrm{cm} / \mathrm{MHz}$, corresponding to a CAP of $241 \mathrm{~dB} / \mathrm{m}$, can accurately diagnose liver steatosis.

ORCID: Pyeong Hwa Kim: https://orcid.org/0000-0003-4276-8803; Young Ah Cho: https://orcid.org/0000-0001-6722-121X; Hee Mang Yoon: https://orcid.org/00000001-6491-5734; Boram Bak: https://orcid.org/0000-0003-0409-6292; Jin Seong Lee: https://orcid.org/0000-0002-8470-4595; Ah Young Jung: https://orcid.org/00000002-7427-6240; Seak Hee Oh: https://orcid.org/0000-0002-9672-8877; Kyung Mo Kim: https://orcid.org/0000-0001-7896-6751

\section{Author Contributions}

Conceptualization: Kim PH, Cho YA, Yoon HM, Kim KM. Data acquisition: Kim PH, Bak B. Data analysis or interpretation: Kim PH, Cho YA, Yoon HM, Lee JS, Jung AY, Oh SH, Kim KM. Drafting of the manuscript: Kim PH, Cho YA. Critical revision of the manuscript: Kim PH, Cho YA, Yoon HM, Bak B, Lee JS, Jung AY, Oh SH, Kim KM. Approval of the final version of the manuscript: all authors.

\section{Conflict of Interest}

No potential conflict of interest relevant to this article was reported.

\section{References}

1. Schwimmer JB, Deutsch R, Kahen T, Lavine JE, Stanley C, Behling C. Prevalence of fatty liver in children and adolescents. Pediatrics 2006;118:1388-1393.

2. Ortiz-Lopez C, Lomonaco R, Orsak B, Finch J, Chang Z, Kochunov $V G$, et al. Prevalence of prediabetes and diabetes and metabolic profile of patients with nonalcoholic fatty liver disease (NAFLD). Diabetes Care 2012;35:873-878.

3. Vajro P, Lenta S, Socha P, Dhawan A, McKiernan P, Baumann U, et al. Diagnosis of nonalcoholic fatty liver disease in children and adolescents: position paper of the ESPGHAN Hepatology Committee. J Pediatr Gastroenterol Nutr 2012;54:700-713.

4. Feldstein $A E$, Charatcharoenwitthaya $P$, Treeprasertsuk S, Benson $J T$, Enders FB, Angulo P. The natural history of non-alcoholic fatty liver disease in children: a follow-up study for up to 20 years. Gut 2009:58:1538-1544.

5. Adams $L A$, Anstee QM, Tilg $H$, Targher $G$. Non-alcoholic fatty liver disease and its relationship with cardiovascular disease and other extrahepatic diseases. Gut 2017;66:1138-1153.

6. Pinyopornpanish K, Khoudari G, Saleh MA, Angkurawaranon C, Pinyopornpanish $\mathrm{K}$, Mansoor $\mathrm{E}$, et al. Hepatocellular carcinoma in nonalcoholic fatty liver disease with or without cirrhosis: a population-based study. BMC Gastroenterol 2021;21:394.

7. Stine JG, Wentworth BJ, Zimmet A, Rinella ME, Loomba R, Caldwell $\mathrm{SH}$, et al. Systematic review with meta-analysis: risk of hepatocellular carcinoma in non-alcoholic steatohepatitis without cirrhosis compared to other liver diseases. Aliment Pharmacol Ther 2018:48:696-703.

8. Lee SS, Park SH. Radiologic evaluation of nonalcoholic fatty liver disease. World J Gastroenterol 2014;20:7392-7402.

9. Sasso M, Audiere S, Kemgang A, Gaouar F, Corpechot C, Chazouilleres $\mathrm{O}$, et al. Liver steatosis assessed by controlled attenuation parameter (CAP) measured with the $\mathrm{XL}$ probe of the FibroScan: a pilot study assessing diagnostic accuracy. Ultrasound Med Biol 2016;42:92-103.

10. Eddowes PJ, Sasso M, Allison M, Tsochatzis E, Anstee QM, Sheridan $D$, et al. Accuracy of FibroScan controlled attenuation parameter and liver stiffness measurement in assessing steatosis and fibrosis in patients with nonalcoholic fatty liver disease. Gastroenterology 
2019;156:1717-1730.

11. Ferraioli G, Calcaterra V, Lissandrin R, Guazzotti M, Maiocchi L, Tinelli $C$, et al. Noninvasive assessment of liver steatosis in children: the clinical value of controlled attenuation parameter. BMC Gastroenterol 2017;17:61.

12. Shin J, Kim MJ, Shin HJ, Yoon H, Kim S, Koh H, et al. Quick assessment with controlled attenuation parameter for hepatic steatosis in children based on MRI-PDFF as the gold standard. BMC Pediatr 2019;19:112.

13. Kimura S, Tanaka K, Oeda S, Inoue K, Inadomi C, Kubotsu Y, et al. Effect of skin-capsular distance on controlled attenuation parameter for diagnosing liver steatosis in patients with nonalcoholic fatty liver disease. Sci Rep 2021;11:15641.

14. Tada T, lijima H, Kobayashi N, Yoshida M, Nishimura T, Kumada T, et al. Usefulness of attenuation imaging with an ultrasound scanner for the evaluation of hepatic steatosis. Ultrasound Med Biol 2019;45:2679-2687.

15. Bae JS, Lee DH, Lee JY, Kim H, Yu SJ, Lee JH, et al. Assessment of hepatic steatosis by using attenuation imaging: a quantitative, easy-to-perform ultrasound technique. Eur Radiol 2019;29:64996507.

16. Jang JK, Kim SY, Yoo IW, Cho YB, Kang HJ, Lee DH. Diagnostic performance of ultrasound attenuation imaging for assessing lowgrade hepatic steatosis. Eur Radiol 2022;32:2070-2077.

17. von Elm E, Altman DG, Egger M, Pocock SJ, Gotzsche PC, Vandenbroucke JP, et al. Strengthening the Reporting of Observational Studies in Epidemiology (STROBE) statement: guidelines for reporting observational studies. BMJ 2007;335:806808.

18. Sasso $M$, Beaugrand $M$, de Ledinghen $V$, Douvin $C$, Marcellin $P$, Poupon $R$, et al. Controlled attenuation parameter (CAP): a novel VCTE guided ultrasonic attenuation measurement for the evaluation of hepatic steatosis: preliminary study and validation in a cohort of patients with chronic liver disease from various causes. Ultrasound Med Biol 2010;36:1825-1835.

19. Lee DH. Quantitative assessment of fatty liver using ultrasound attenuation imaging. J Med Ultrason (2001) 2021;48:465-470.

20. Yi DY, Kim SC, Lee JH, Lee EH, Kim JY, Kim YJ, et al. Clinical practice guideline for the diagnosis and treatment of pediatric obesity: recommendations from the Committee on Pediatric Obesity of the Korean Society of Pediatric Gastroenterology Hepatology and Nutrition. Korean J Pediatr 2019;62:3-21.

21. Vos MB, Abrams SH, Barlow SE, Caprio S, Daniels SR, Kohli R, et al. NASPGHAN clinical practice guideline for the diagnosis and treatment of nonalcoholic fatty liver disease in children: recommendations from the Expert Committee on NAFLD (ECON) and the North American Society of Pediatric Gastroenterology, Hepatology and Nutrition (NASPGHAN). J Pediatr Gastroenterol Nutr 2017;64:319-334.
22. Middleton MS, Van Natta ML, Heba ER, Alazraki A, Trout AT, Masand $P$, et al. Diagnostic accuracy of magnetic resonance imaging hepatic proton density fat fraction in pediatric nonalcoholic fatty liver disease. Hepatology 2018;67:858-872.

23. Kleiner DE, Brunt EM, Van Natta M, Behling C, Contos MJ, Cummings $\mathrm{OW}$, et al. Design and validation of a histological scoring system for nonalcoholic fatty liver disease. Hepatology 2005;41:1313-1321.

24. Rutjes AW, Reitsma JB, Coomarasamy A, Khan KS, Bossuyt PM. Evaluation of diagnostic tests when there is no gold standard: a review of methods. Health Technol Assess 2007;11:iii-X, 1-51.

25. Ferraioli G, Tinelli C, De Silvestri A, Lissandrin R, Above E, Dellafiore $C$, et al. The clinical value of controlled attenuation parameter for the noninvasive assessment of liver steatosis. Liver Int 2016;36:1860-1866.

26. Colton T. Statistic in medicine. Boston, MA: Little, Brown and Company, 1974.

27. Ferraioli G, Maiocchi L, Savietto G, Tinelli C, Nichetti M, Rondanelli $M$, et al. Performance of the attenuation imaging technology in the detection of liver steatosis. J Ultrasound Med 2021;40:1325-1332.

28. Jang JK, Choi SH, Lee JS, Kim SY, Lee SS, Kim KW. Accuracy of the ultrasound attenuation coefficient for the evaluation of hepatic steatosis: a systematic review and meta-analysis of prospective studies. Ultrasonography 2022;41:83-92.

29. D'Hondt A, Rubesova E, Xie H, Shamdasani V, Barth RA. Liver fat quantification by ultrasound in children: a prospective study. AJR Am J Roentgenol 2021;217:996-1006.

30. Brody AS, Frush DP, Huda W, Brent RL; American Academy of Pediatrics Section on Radiology. Radiation risk to children from computed tomography. Pediatrics 2007;120:677-682.

31. Frush DP, Donnelly LF, Rosen NS. Computed tomography and radiation risks: what pediatric health care providers should know. Pediatrics 2003;112:951-957.

32. Jeon SK, Lee JM, Joo I. Clinical feasibility of quantitative ultrasound imaging for suspected hepatic steatosis: intra- and inter-examiner reliability and correlation with controlled attenuation parameter. Ultrasound Med Biol 2021;47:438-445.

33. Myers RP, Pomier-Layrargues $G$, Kirsch R, Pollett A, Duarte-Rojo $A$, Wong $D$, et al. Feasibility and diagnostic performance of the FibroScan XL probe for liver stiffness measurement in overweight and obese patients. Hepatology 2012;55:199-208.

34. Yang A, Nguyen M, Ju I, Brancatisano A, Ryan B, van der Poorten D. Utility of Fibroscan $X L$ to assess the severity of non-alcoholic fatty liver disease in patients undergoing bariatric surgery. Sci Rep 2021;11:14006.

35. Shen F, Zheng RD, Shi JP, Mi YQ, Chen GF, Hu X, et al. Impact of skin capsular distance on the performance of controlled attenuation parameter in patients with chronic liver disease. Liver Int 2015;35:2392-2400. 
36. Mandelia C, Kabbany MN, Worley S, Conjeevaram Selvakumar PK. Performance characteristics, intra- and inter-operator agreement of transient elastography in pediatric nonalcoholic fatty liver disease. J Pediatr Gastroenterol Nutr 2021;72:430-435.

37. Li L, Liu DW, Yan HY, Wang ZY, Zhao SH, Wang B. Obesity is an independent risk factor for non-alcoholic fatty liver disease: evidence from a meta-analysis of 21 cohort studies. Obes Rev 2016;17:510-519.

38. Hawkins DM, Garrett JA, Stephenson B. Some issues in resolution of diagnostic tests using an imperfect gold standard. Stat Med 2001;20:1987-2001. 